\title{
Pneumococcal Meningitis in a Child With Sickle Cell Anemia: A Case Report
}

\author{
Demetrio Kiriazopulos ${ }^{\mathrm{a}, \mathrm{d}}$, Palmino Pedroni ${ }^{\mathrm{b}}$, Guya Occhic ${ }^{\mathrm{c}}$, Giovanna Sassi ${ }^{\mathrm{a}}$, Angela Corna ${ }^{\mathrm{a}}$, \\ Franca Cieria, Eugenio Cipolletta ${ }^{a}$, Marinetta Orobello ${ }^{a}$, Mario Colombo ${ }^{a}$
}

\begin{abstract}
Children with sickle cell disease are mainly exposed to various infections, among which there is bacterial meningitis. We present a brief report of a pneumococcal meningitis, due to Streptococcus pneumoniae $33 \mathrm{~F}$, in a 3-year-old boy of African race with sickle cell anemia. He was vaccinated against Streptococcus pneumoniae with 13-valent vaccine, and he was also on antibiotic prophylaxis. The child has been successfully treated with cephalosporins for a complete duration of 6 weeks, while hospitalization lasted about 3 weeks. Ceftriaxone was replaced by cefotaxime to avoid risk of hemolysis, as we found in literature. During recovery, the little boy presented with osteomyelitis, so vancomycin was added to therapy with good results. Finally, neurosensorial deafness was found, thus the child was quickly directed to cochlear implant intervention. This case report revealed that sometimes pneumococcal vaccination and antibiotic prophylaxis are not enough to prevent pneumococcal meningitis in children with sickle cell disease. Moreover, we noticed that cefotaxime avoids autoimmune hemolysis, which may be fatal to anemic children, while vancomycin may help to prevent complications such as osteomyelitis.
\end{abstract}

Keywords: Sickle cell anemia; Pneumococcal meningitis; Pneumococcal vaccine; Streptococcus pneumoniae 33F; Osteomyelitis; Autoimmune hemolysis

\section{Introduction}

Sickle cell anemia or drepanocytosis is a hereditary genetic blood disorder, which can manifest itself in different levels of

\footnotetext{
Manuscript accepted for publication September 29, 2015

aDepartment of Pediatrics and Neonatology, Hospital of Manerbio, AO Desenzano del Garda, Brescia, Italy

bService of Microbiology, Hospital of Manerbio, AO Desenzano del Garda, Brescia, Italy

'Service of Child Neuropsychiatry, AO Desenzano del Garda, Brescia, Italy

${ }^{\mathrm{d} C}$ Corresponding Author: Demetrio Kiriazopulos, Department of Pediatrics and Neonatology, Hospital of Manerbio, Manerbio 25025, Brescia, Italy.

Email: d.kiriazopulos@gmail.com
}

doi: http://dx.doi.org/10.14740/ijcp225w severity. People who suffer from this pathology are exposed to various diseases and infections because of poorly oxygenated blood, which does not feed suitably the tissues and the organs. Concerning infections, subjects with sickle cell disease, especially young ones, have a higher probability to develop bacterial meningitis. The most common pathogen of these is Streptococcus pneumoniae also called pneumococcus [1].

In Italy, according to the last report of the "National Center for Epidemiology, Surveillance and Prevention of Health" (March 2015), pneumococcus alone is responsible for about $33 \%$ of all meningitis cases, $15 \%$ of which involve children under 5 years.

\section{Case Report}

\section{History}

A 3-year-old boy, born in Italy to Nigerian parents, was brought to the Pediatric Emergency Department with high fever and drowsiness for 2 days.

The child was suffering from sickle cell anaemia like his father, and he was followed in another center. According to their protocol, he was undergoing antibiotic prophylaxis with amoxicillin [2].

He had received all recommended vaccinations and also some that are optional, such as anti-pneumococcal and antimeningococcal.

\section{Physical examination}

Physical examination revealed high temperature $\left(102.2{ }^{\circ} \mathrm{F} / 39\right.$ ${ }^{\circ} \mathrm{C}$ ) with weakness condition, typical fetal position with neck stiffness and pain in the spine, while diffuse crackles were audible over both lung fields $\left(97 \% \mathrm{SO}_{2}, 140 \mathrm{bpm}\right)$. Also a mild splenomegaly was found, compatible with the underlying disease.

\section{Diagnostic and therapeutic approach}

In suspicion of meningitis, the child was immediately transferred to the Department of Pediatrics where urgent blood ex- 
ams with bacterial culture, urinalysis, blood gas analysis and instrumental tests, like fundus oculi and brain CT scan, were requested and performed. Having fundus oculi excluded signs of intracranial hypertension, a lumbar puncture was performed for the chemical-physical examination and the microbiological culture of cerebrospinal fluid (CSF).

In consideration of the clinical presentation at ER, paracetamol, saline infusion and dexamethasone $(0.6 \mathrm{mg} / \mathrm{kg} /$ day $)$ were administered and an empirical antibiotic therapy was fixed with ceftriaxone $(100 \mathrm{mg} / \mathrm{kg} /$ day $)$ [3] .

Within a few hours the child seemed clinically more responsive. In the meantime, the first results became available: the blood count showed low levels of hemoglobin $(6.5 \mathrm{~g} / \mathrm{dL})$, low levels of red blood cells $(2,420,000 / \mu \mathrm{L})$, low hematocrit $(19 \%)$ and neutrophilia (80.7\%), while white blood cells were within the limits $(13,200 / \mu \mathrm{L})$, platelets also $(254,000 / \mu \mathrm{L})$. There was high C-reactive protein $(34.1 \mathrm{mg} / \mathrm{dL})$. The bacteriological examination of CSF showed the presence of grampositive diplococci, so vancomycin was added to ceftriaxone, pending culture result with antibiogram [4].

Blood glucose and electrolytes were normal, and saline solution was replaced with a physiological balanced electrolyte solution for children. Brain CT scan documented a dilatation of the left lateral ventricle without signs of focal injuries. Sleep-deprived EEG was normal.

After 3 days the blood culture performed at admission was negative, as well as the nasal swab and the throat one. The bacterial culture of CSF reported positive for Streptococcus pneumoniae, sensitive to ceftriaxone and the identification of the bacterial strain showed serotype $33 \mathrm{~F}$ to be responsible for the meningitis. The diagnosis was pneumococcal meningitis; hence vancomycin was suspended.

Then an unusual lower value of hemoglobin $(4.7 \mathrm{~g} / \mathrm{dL})$ was disclosed through routine blood tests. The child was consequently transfused with a bag of packed red blood cells adopting the proper precautions.

The fourth day, dexamethasone was progressively reduced and then finally stopped on the fifth day, instead ceftriaxone was replaced with cefotaxime, having found many publications about the risk of severe immune hemolysis in patients with sickle cell disease treated with ceftriaxone [5].

All the following checks of hemoglobin levels showed values above $8.0 \mathrm{~g} / \mathrm{dL}$.

On the seventh day, the child presented fever and elevation of inflammatory markers (ESR and C-reactive protein) associated with sudden onset of walking difficulty. He felt hip pain only during active or passive moving of the right lower limb, but he was smiling at rest. Therefore, excluding a vasoocclusive crisis, in clinical suspicion of osteomyelitis (even $\mathrm{X}$-ray was negative), vancomycin was administered for five more days until reduction of inflammatory markers and normal pain-free hip mobility were noticed [6].

Gradually the clinical conditions of the little patient and laboratory parameters improved.

Towards the end of hospitalization, bilateral hearing loss was diagnosed by otoacoustic emissions (OAEs) and brainstem auditory evoked potentials (BAEPs).

During his hospital stay, the child was repeatedly visited by neuropsychiatrists, who perceived the progressive improve- ment in psychomotricity.

The little boy was finally discharged after 23 days of hospitalization. As home therapy, oral cefaclor was prescribed for 3 weeks. Cephalosporins were overall administered for 6 weeks ( 3 weeks intravenously and 3 weeks orally).

Furthermore, he was scheduled for a cochlear implant in a specialized center.

\section{Discussion}

The child had previously undergone pneumococcal vaccination with three doses of 13-valent vaccine "Prevenar 13" (PCV13), he also was in antibiotic prophylaxis with amoxicillin, but despite this he got sick. Two doses of the vaccine have been given between 7 and 11 months and the recall dose around 15 months, according to the national vaccination schedule in use. "Prevenar 13" includes the seven serotypes of the vaccine "Prevenar 7" (4, 6B, 9V, 14, 18C, 19F and 23F) and it presents six additional serotypes $(1,3,5,6 \mathrm{~A}, 7 \mathrm{~F}$ and 19A). These 13 serotypes represent the most worldwide common pathogenic strains in young children, but nowadays about 90 serotypes of Streptococcus pneumoniae are known, all potentially responsible for invasive infections.

The serotype that caused meningitis in our little patient was $33 \mathrm{~F}$, which is not present in the administered vaccine. Currently a pneumococcal vaccine that contains serotype $33 \mathrm{~F}$ is available on the market, and it is the 23 -valent vaccine (PPSV23), which comprises a total of 23 strains [7].

Moreover, it cannot be verified if parents, at their home, have given the antibiotic in the appropriate way and every time.

The patient during pharmacological treatment had a sudden and further reduction of hemoglobin levels. May have this been a case of a hematological crisis due to ceftriaxone? We cannot say it with certainty, but in literature we have indeed found that if patients with sickle cell disease, stricken by pneumococcal meningitis, are treated with ceftriaxone (as required by general protocols on meningitis), they probably may present severe autoimmune hemolytic anemia thus aggravating their situation. In such cases, for us, it is preferable to switch to another cephalosporin as cefotaxime, avoiding any risk [8].

Besides, as you can see from the case discussed, an early and correct antibiotic therapy may not always avoid disseminations. The child, although immediately and continuously treated with a cephalosporin (ceftriaxone first and then cefotaxime), has probably had osteomyelitis as complication, requiring a double antibiotic therapy with a high diffusibility glycopeptide (vancomycin) associated to cephalosporin [9].

This may have been favored by the relative immunodeficiency of the subject.

At last, the child presented neurosensorial hearing loss despite early and adequate therapy. Neurosensorial hearing loss, as reported by Nelson's Textbook, "is the most common consequence of bacterial meningitis and it is often already present at the time of admission to ER. It is usually caused by infection and it's found in $30 \%$ of patients with pneumococcal meningitis" [10]. 


\section{Conclusion}

In light of the discussion of this case, we can draw the following considerations. 1) In order to better prevent pneumococcal disease in children with sickle cell disease, it may be suitable to use the 23-valent pneumococcal vaccine. 2) To treat pneumococcal meningitis in a patient with sickle cell anemia, it is advisable to use a cephalosporin such as cefotaxime and not ceftriaxone, since this one can sometimes cause severe autoimmune hemolysis. 3) To prevent the occurrence of dissemination, it is useful to immediately undertake, in these children, an antibiotic therapy with a cephalosporin (cefotaxime) associated with a high diffusibility glycopeptide (vancomycin). 4) In regard to neurosensorial deafness, which is among the most frequent and inevitable complications, it is very important for an early diagnosis to allow an eventual cochlear implant, which should be realized as soon as possible, before complete cochlear ossification.

The child is fine now, and his psychomotricity develops appropriately for his age. He is currently attending kindergarten, and he is continuously followed by the hematology center. $\mathrm{He}$ is also bearer of a cochlear implant and so he is monitored by the audiology center.

\section{Conflict of Interest}

The authors declare that there is no conflict of interests regarding the publication of this paper.

\section{References}

1. Wong WY. Prevention and management of infection in children with sickle cell anaemia. Paediatr Drugs. 2001;3(11):793-801.
2. Hord J, Byrd R, Stowe L, Windsor B, Smith-Whitley K. Streptococcus pneumoniae sepsis and meningitis during the penicillin prophylaxis era in children with sickle cell disease. J Pediatr Hematol Oncol. 2002;24(6):470-472.

3. Borchorst S, Moller K. The role of dexamethasone in the treatment of bacterial meningitis - a systematic review. Acta Anaesthesiol Scand. 2012;56(10):1210-1221.

4. Le Saux N. Guidelines for the management of suspected and confirmed bacterial meningitis in Canadian children older than one month of age. Paediatr Child Health. 2014;19(3):141-152.

5. Goyal M, Donoghue A, Schwab S, Hasbrouck N, Khojasteh S, Osterhoudt K. Severe hemolytic crisis after ceftriaxone administration. Pediatr Emerg Care. 2011;27(4):322-323.

6. Huang WL, Wuz TM, Yu CK, Cheng YL, Lien C. Pneumococcal osteomyelitis: report of one case. Pediatr Neonatol. 2008;49(6):248-253.

7. Adamkiewicz TV, Sarnaik S, Buchanan GR, Iyer RV, Miller ST, Pegelow CH, Rogers ZR, et al. Invasive pneumococcal infections in children with sickle cell disease in the era of penicillin prophylaxis, antibiotic resistance, and 23 -valent pneumococcal polysaccharide vaccination. J Pediatr. 2003;143(4):438-444.

8. Quillen K, Lane C, Hu E, Pelton S, Bateman S. Prevalence of ceftriaxone-induced red blood cell antibodies in pediatric patients with sickle cell disease and human immunodeficiency virus infection. Pediatr Infect Dis J. 2008;27(4):357-358.

9. Hameed N, Tunkel AR. Treatment of Drug-resistant Pneumococcal Meningitis. Curr Infect Dis Rep. 2010;12(4):274-281.

10. Wasier AP, Chevret L, Essouri S, Durand P, Chevret S, Devictor D. Pneumococcal meningitis in a pediatric intensive care unit: prognostic factors in a series of 49 children. Pediatr Crit Care Med. 2005;6(5):568-572. 